\title{
Improved immunohistochemical evaluation of micrometastases in sentinel lymph nodes of cutaneous melanoma with 'MCW Melanoma Cocktail' - A mixture of monoclonal antibodies to MART-I, melan-A, and tyrosinase
} Vinod B Shidham*, Dan Qi, R Nagarjun Rao, Scott M Acker, ChungChe Chang, Bal Kampalath, Glen Dawson, Jinobya K Machhi and Richard A Komorowski

Address: Department of Pathology, Medical College of Wisconsin, Milwaukee, WI, USA

Email: Vinod B Shidham* - vshidham@mcw.edu; Dan Qi - dy.di@hosp.wisc.edu; R Nagarjun Rao - arao@mcw.edu; Scott M Acker - scottacker@webtv.net; Chung-Che Chang - jcchang@mcw.edu; Bal Kampalath - bkampala@mcw.edu; Glen Dawson - gdawson@wi.rr.com; Jinobya K Machhi - jinobya@aol.com; Richard A Komorowski - rkomor@mcw.edu

* Corresponding author

Published: 7 May 2003

BMC Cancer 2003, 3:15
Received: 24 January 2003

Accepted: 7 May 2003

This article is available from: http://www.biomedcentral.com/I47I-2407/3//5

(C) 2003 Shidham et al; licensee BioMed Central Ltd. This is an Open Access article: verbatim copying and redistribution of this article are permitted in all media for any purpose, provided this notice is preserved along with the article's original URL.

\begin{abstract}
Background: MART-I, Melan-A, and Tyrosinase have shown encouraging results for evaluation of melanoma micrometastases in sentinel lymph nodes, as compared to conventionally used S- 100 protein and HMB-45. To achieve higher sensitivity, some studies recommend evaluation of three sections, each at intervals of $200 \mu$. This would mean, routine staining of three adjacent sections in each of the three clusters at intervals of $200 \mu$, requiring nine slides resulting in added expense. If a cocktail of these antibodies could be used, only one section would be required instead of three generating significant cost savings.
\end{abstract}

Methods: We prepared a combination of monoclonal antibodies to these three immunomarkers in optimized dilutions (MART-I, clone M2-7CI0, dilution I:500; Melan-A, clone AI03, dilution I:I00; and Tyrosinase, clone T3II, dilution I:50) and designated it as 'MCW melanoma cocktail'. Formalin-fixed paraffin-embedded tissue sections of sentinel lymph nodes from patients with cutaneous melanoma, without macro-metastases were evaluated with this cocktail.

Results: Melanoma micrometastases were easily detectable with the cocktail in 4 I out of 188 slices $(8 / 24$ cases). The diagnostic accuracy amongst five pathologists did not show statistically significant difference. Out of 188 slices, 78 had adjacent sections immunostained individually with MART-I and Melan-A during our previous study. Of these 78 slices, 2 I were positive for melanoma micrometastases with MART-I and Melan-A individually. However, the adjacent section of these slices immunostained with the cocktail detected metastases in four additional slices. Thus, MARTI and Melan-A could not detect melanoma micrometastases individually in 16\% (4/25) of slices positive with the cocktail. Benign capsular nevi were immunoreactive for the cocktail in $4.8 \%$ (9/ 
I88) slices. All $8 \mathrm{I}$ slices of negative test controls (sentinel lymph nodes of mammary carcinoma) were interpreted correctly as negative for melanoma micrometastases.

Conclusions: The melanoma cocktail facilitated easy interpretation of melanoma micrometastases in sentinel lymph nodes with high interobserver agreement. There was improvement in detection rate with the cocktail as compared to MART-I and Melan-A individually. Furthermore, this approach facilitates cost savings.

\section{Background}

Lymphadenectomies with routine histopathologic evaluation have been performed for staging and regional control of primary cutaneous melanoma, even in cases without clinical evidence of metastasis. Because of significant morbidity associated with the procedure, regional lymphadenectomy has been generally recommended only for patients with evidence of regional lymph node involvement [1]. To assess the involvement of regional lymph nodes, the biopsies of sentinel lymph nodes are invaluable for detecting occult metastases without the significant morbidity associated with lymphadenectomy [28]. Previous studies have reported that the status of sentinel lymph node is an accurate reflection of the regional lymph node status as a whole and that treatment decisions can be made based on the results of sentinel lymph nodes alone [9-11].

Previously, we had demonstrated higher diagnostic accuracy in detecting and diagnosing melanoma micrometastases in sentinel lymph node using monoclonal antibodies to MART-1 (clone M2-7C10) and Melan-A (clone A103) in comparison to the traditionally used immunohistochemical markers, S-100 protein (S-100) and HMB-45 [6]. The cytoplasmic staining with these immunomarkers does not obscure the nuclear details and facilitates accurate interpretation of tumor cells, macrophages, and nevus cells in contrast to S-100 [6,12]. A monoclonal antibody to a third melanoma immunomarker, Tyrosinase (clone T311), showed an immunostaining pattern similar to MART-1 and Melan-A [13].

In the present study, we evaluated the combination of monoclonal antibodies; MART-1, Melan-A, and Tyrosinase in optimized titers (Table 1) for evaluation of sentinel lymph nodes of cutaneous melanoma. We designated this combination after our institution as 'MCW melanoma cocktail' $[6,14]$. Hypothetically, due to the immunoreactivity of the cocktail towards three epitopes of two antigens in the same tissue section, combined use of these immunomarkers should increase the sensitivity while still maintaining their individual high specificity. The possibility of using a cocktail of these monoclonal antibodies for the evaluation of melanoma sentinel lymph node would require only one section as compared to three ad- jacent sections for each of the immunomarker, with resultant cost savings of approximately two thirds.

The aim of this study was to evaluate the accuracy of detecting and interpreting micrometastases in the sentinel lymph nodes of patients with cutaneous melanoma utilizing this cocktail in a single section instead of three adjacent sections needing each of the three immunomarkers in the cocktail. We also compared the cocktail against MART-1 and Melan-A individually.

\section{Methods}

Sentinel lymph node specimens were grossed as described previously [6]. Formalin-fixed paraffin-embedded tissue sections of 269 slices were evaluated with the cocktail (Figure 1). In some cases more than one lymph nodes were submitted as sentinel lymph nodes. The fresh, unfixed sentinel lymph nodes were transected into slices no thicker than $3 \mathrm{~mm}$ across the long axis from one pole to the other. They were fixed flat in formalin after wrapping them in tissue paper to avoid curling and were processed for paraffin embedding.

The test group included 188 slices of 88 sentinel lymph nodes ( 3 to 6 slices per lymph node) from 24 cases ( 1 to 4 lymph nodes per case) of AJCC stage I and II cutaneous melanoma lacking metastases which were easily detectable in sections stained with hematoxylin and eosin. These lymph nodes were from various regions other than the axillary areas. A negative test control group was studied to verify the specificity of the cocktail. It consisted of 81 slices of 30 sentinel lymph nodes ( 3 to 5 slices per lymph node) from the axillary area in 30 cases of mammary carcinoma, of which 40 slices were positive for mammary carcinoma metastases (Figure 1).

All the sections in the test group and negative control group were immunostained with the cocktail (the details of antibodies in the cocktail are shown in Table 1). As a standardization process to confirm positive immunoreactivity, 35 melanomas (including one spindle cell melanoma and one clear cell melanoma) were evaluated. All 35 melanomas were immunoreactive with the cocktail. Antibodies from a few additional sources, other than those mentioned in Table 1, were also evaluated. However, only the antibodies from the sources mentioned in Table 1 


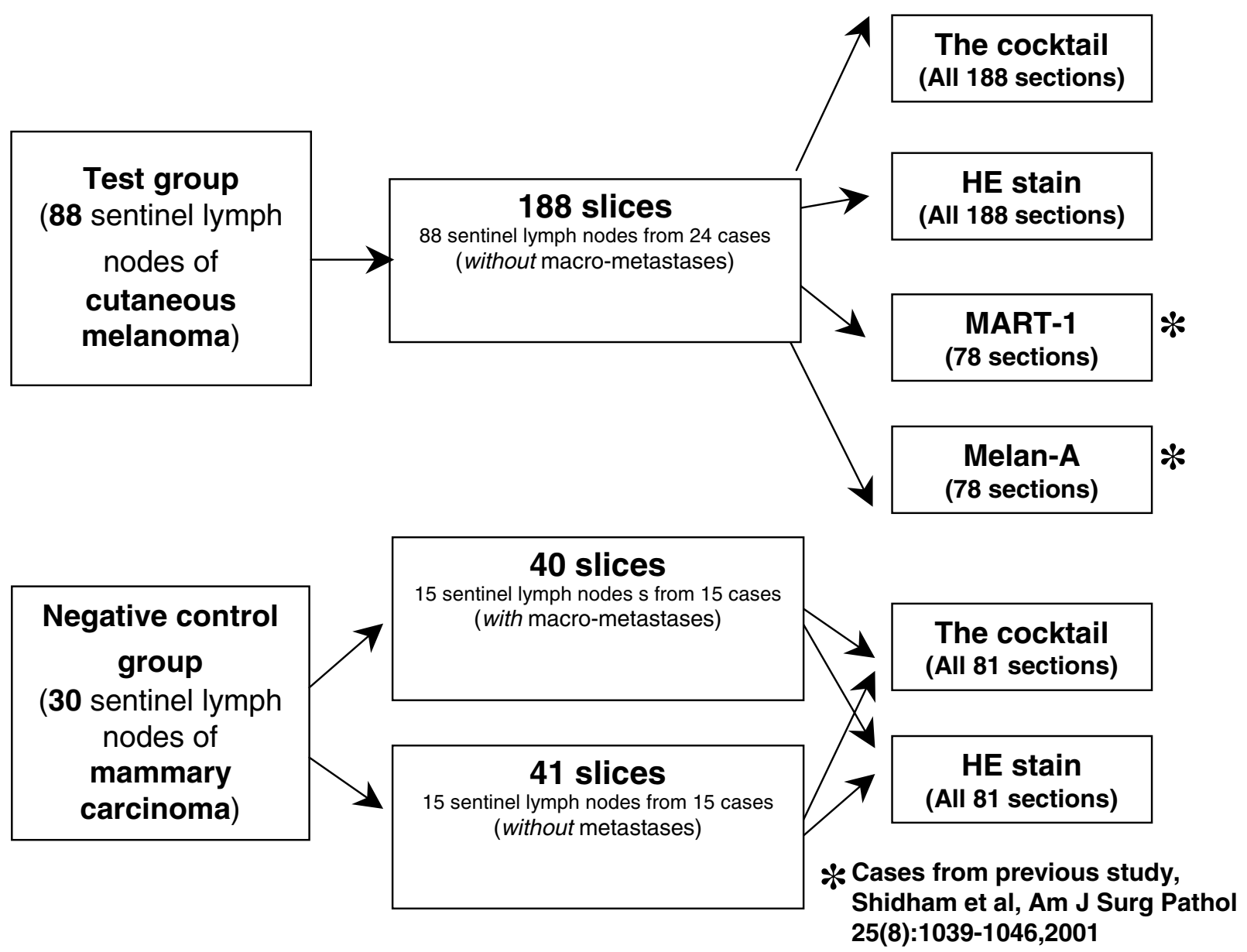

Figure I

Algorithm showing the processing of sections of sentinel lymph nodes (HE, hematoxylin and eosin). *Immediately adjacent level to these sections during previous study [Ref\# [27]] were immunostained with the cocktail during this study.

Table I: Details of the monoclonal antibodies in the 'MCW Melanoma Cocktail'.

\begin{tabular}{llll}
\hline Marker & Clone & Source & *Final Dilution in the cocktail \\
\hline MART-I & M2-7C10 & Signet Laboratories, Inc. Dedham, MA & $1: 500$ \\
Melan-A & Al03 & Dako Corporation, Carpinteria, CA & I:100 \\
Tyrosinase & T3II & Novocastra Laboratories Ltd Newcastle upon Tyne, UK & I:50
\end{tabular}

* Optimum dilution for each antibody was standardized individually for that batch of antibodies with the sections of known melanoma positive control. The standardized dilution was achieved as final titer in the cocktail by adding $20 \mu \mathrm{l}$ MART-I, $100 \mu \mathrm{l} \mathrm{Melan-A}$, and $200 \mu \mathrm{l} \mathrm{Tyrosinase} \mathrm{to} 9.68 \mathrm{ml}$ of DAKO Antibody diluent (Dako Corporation, Carpinteria, CA).

with heat antigen retrieval protocol achieved optimum results without significant background staining.
The sections were pretreated for antigen retrieval using 'Dako pH 6.0 target retrieval solution' ${ }^{1}$ (Dako Corporation, Carpinteria, CA) at $95-100^{\circ} \mathrm{C}$ for 35 minutes and cooled at room temperature for 20 minutes. The target re- 
Table 2: Evaluation of interobserver reproducibility in 188 sections.

\begin{tabular}{llll}
\hline Marker & Observer & Az & P value* \\
\hline & A & 0.8525 & .0743 \\
MCW & B & 0.9268 & \\
melanoma & C & 0.9205 & \\
cocktail & D & 0.9487 & 1.0000 \\
& E & 1.000 & \\
\hline
\end{tabular}

Az, Area under ROC curves. * Mann-Whitney statistics

trieval solution must be at a temperature of $95-100^{\circ} \mathrm{C}$ from the start. The duration of this heat antigen retrieval step was crucial for avoiding non-specific background staining. It was optimal for all the three antibodies in the cocktail.

Three-micron thick serial sections of paraffin-embedded tissue blocks were numbered consecutively from 1 to 3 . Section \# 1 was stained with hematoxylin and eosin. Section \#2 was immunostained with 'melanoma cocktail' using Diaminobenzidine Hydrochloride (DAB) as a chromogen. Section \#3 was used as a negative control without primary antibody. A positive control consisting of a formalin-fixed paraffin-embedded tissue section of melanoma demonstrating reactivity for the cocktail was processed with each batch of slides. Two adjacent sections were immunostained with MART-1 and Melan-A respectively during our previous study on 78 out of 188 slices (from 14 cases) [6]. The immediately adjacent unstained sections during the previous study were used. They were immunostained with the cocktail in this study.

Five pathologists interpreted all immunostained slides independently to determine the presence of melanoma micrometastases. Whenever indicated, respective adjacent hematoxylin and eosin stained sections and negative control sections were also used to facilitate the final interpretation.

Statistical evaluation for degree of interobserver agreement was based on the Receiver Operating Characteristic (ROC) curve, which takes into consideration the non-binary spectrum of surgical pathology interpretation $[6,15-$ $19]$. For the generation of the ROC curve and to calculate the areas under the ROC curves (Az values), the spectrum of the interpretation was spread into five points with negative and positive at each end [6].

A consensus binary negative or positive diagnosis was reached by all five pathologists in tandem under a multihead microscope. This was used as the final diagnosis for statistical analysis. Az values by different observers for 188 sections were compared using non-parametric methods based on the Mann-Whitney statistics [20]. SAS statistical software (The SAS institute, Cary, NC) was implemented for this statistical analysis (Table 2).

\section{Results}

Of the 188 sections immunostained with 'melanoma cocktail', 41 sections (8/24 cases) were positive for melanoma micrometastases (Figure $2 \mathrm{~A} \& 2 \mathrm{~B}$ ). Az values for the cocktail by all five pathologists were high and ranged from 0.8525 to 1.000 (maximum possible Az value is 1.000) without statistically significant differences ( $\mathrm{p}$ .0743) (Table 2).

In general, the micrometastases were subtle and difficult to detect in the hematoxylin and eosin stained sections alone. Depending on the observer, micrometastases of melanoma in 41 slices were detectable in only $30-45 \%$ of hematoxylin and eosin sections. However, with closer methodical scrutiny, these micrometastases were seen retrospectively in all 41 adjacent sections stained with hematoxylin and eosin. All 81 sections from sentinel lymph nodes of mammary carcinoma in the negative test control group (Figure 1) were interpreted unequivocally as negative for melanoma micrometastases by each of the five pathologists (Figure 2C).

Out of 188 slices, a pool of sections immunostained individually with MART-1 and Melan-A during our previous study (Figure 1) were compared with the cocktail. Melanoma micrometastases were detected in 21 of 78 slices immunostained with MART- 1 and Melan-A individually. However, 25 slices demonstrated micrometastases with the cocktail. These four slices (from 2 lymph nodes of 2 cases), negative for micrometastases with MART- 1 and Melan-A, showed melanoma micrometastases predominantly as single cells. These micrometastases were detectable retrospectively in adjacent sections stained with hematoxylin and eosin in all four slices. Thus, MART-1 and Melan-A individually could not detect micrometastases in $16 \%(4 / 25)$ of the slices. No sections which were negative for micrometastases with the cocktail demon- 


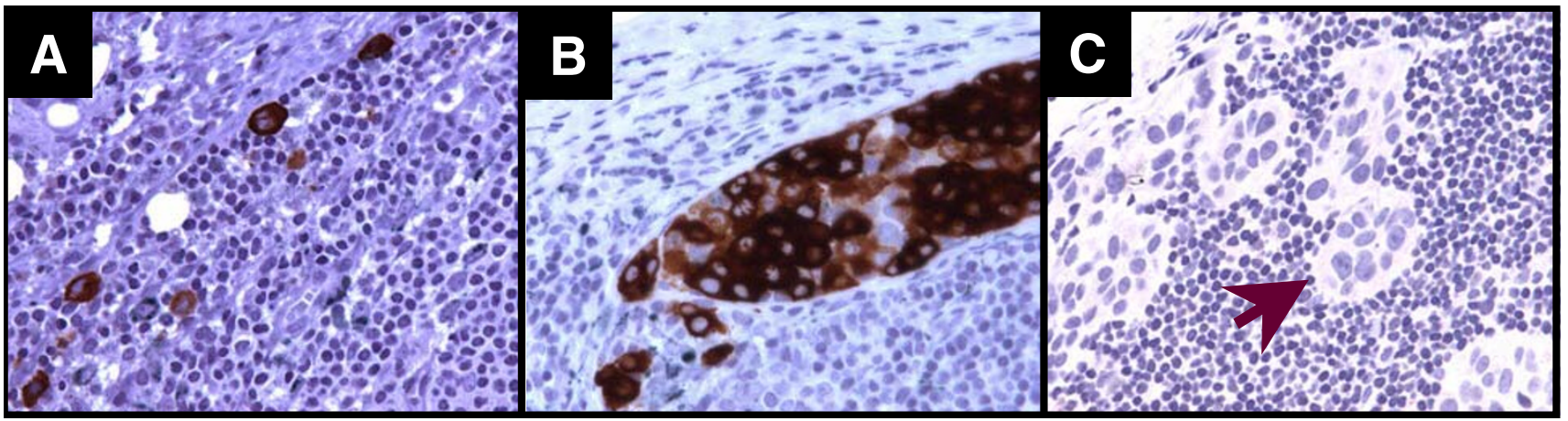

Figure 2

Sentinel lymph node positive for melanoma micrometastases. A- as single cells, B- as groups of cohesive cells, C- Negative control-sentinel lymph node with metastases of mammary carcinoma (arrow). Note the non-immunoreactivity of mammary carcinoma and adjacent lymphoid tissue for the cocktail. (A through C- Immunostaining with hematoxylin counterstain).

strated micrometastases with MART-1 and Melan-A individually.

The 'melanoma cocktail' did not show background staining of non-melanocytic cells in the sentinel lymph nodes (Figure 2 and 3 ) and demonstrated immunostaining pattern identical to MART- 1 and Melan-A reported previously [6]. $5 \%(9 / 188)$ slices from $21 \%(5 / 24)$ of the cases showed groups of cohesive spindle cells which were immunoreactive for the cocktail in the capsule of sentinel lymph nodes. The bland nuclear morphology of these spindle cells was consistent with benign capsular melanocytic nevi (Figure 3). None of the 81 sections of sentinel lymph nodes from 30 cases of mammary carcinoma demonstrated benign capsular melanocytic nevi.

\section{Discussion}

At present histomorphologic examination with immunohistochemistry remains the most reliable and practical means of detecting melanoma micrometastases in sentinel lymph nodes $[6,21]$. MART- 1 and Melan-A are two antibodies to different epitopes of the same antigen and are complementary to each other. As previously reported, MART-1 and Melan-A demonstrated predominantly cytoplasmic staining of melanoma cells [6]. The monoclonal antibody (T311) to tyrosinase, has been reported to demonstrate high sensitivity and specificity for melanoma [13]. It has also demonstrated an immunostaining pattern in sentinel lymph nodes identical to MART-1 and MelanA. We combined these three antibodies as 'MCW melanoma cocktail' (Table 1).

In contrast to S-100, these immunomarkers did not obscure nuclear details. In addition, they did not stain the dendritic cells which are generally prominent in the sentinel lymph nodes after the diagnostic biopsy of a primary lesion. This facilitated unequivocal interpretation of singly scattered melanoma cells which were otherwise impossible to identify in sections immunostained for S-100 due to high noise to signal ratio resulting from numerous S-100 immunoreactive dendritic cells in the background. Thus, a highly sensitive immunomarker such as S-100 is less effective for interpretation of sentinel lymph nodes for melanoma micrometastases (especially when present as single cells) than MART-1 and Melan-A [6]. Certain morphological features help to differentiate melanoma cells and other non-melanoma cells such as S100 immunoreactive dendritic cells in sentinel lymph nodes. However, as demonstrated in our previous study, it is not uncommon to face an equivocal situation when S-100 


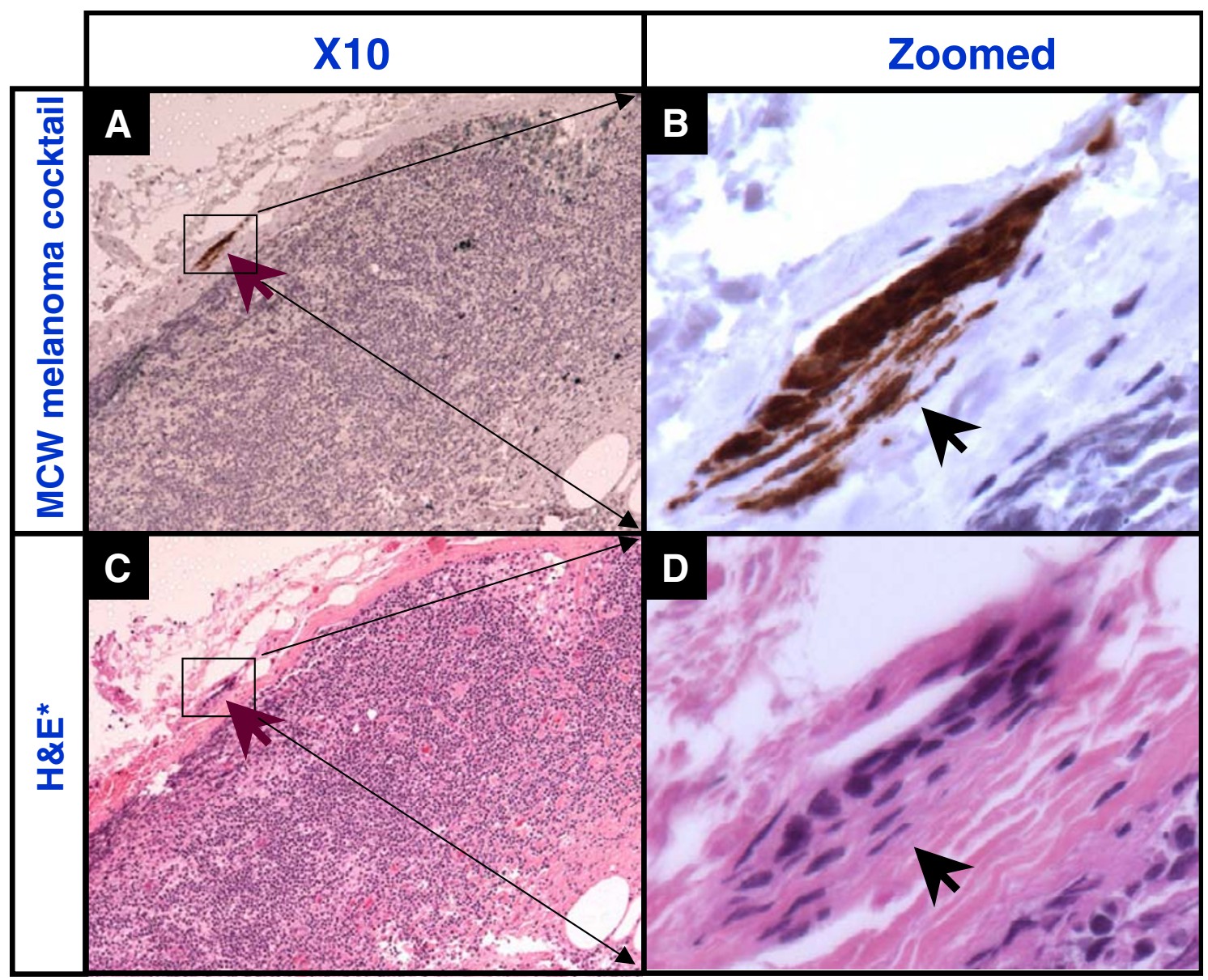

\section{Figure 3}

Capsular nevus (arrows). Groups of bland cohesive spindle cells immunoreactive for the cocktail (A and B) in the capsule of the sentinel lymph node. *Aadjacent section stained with hematoxylin and eosin (C and D).

protein is used as an immunomarker for the interpretation of single melanoma micrometastases in sentinel lymph nodes [6]. Based on our previous study with HMB45 [6] and an initial study with micropthalmia transcription factor [22], these immunomarkers were not considered suitable for inclusion in the cocktail due to the immunoreactivity of non-melanoma cells in the sentinel lymph nodes.

During further evaluation, we confirmed that the single cells immunostained with HMB45 were mast cells and not melanophages. Although not reported in the literature, mast cells in mastocytosis and reactive mast cells in desmoid tumors also showed immunoreactivity for HMB45 with granular cytoplasmic immunostaining pattern. The same cells in the immediately adjacent section were confirmed as mast cell by CD117 (personal experi- ence). Lack of similar staining in the negative control sections excludes their non-specific nature. Mast cells are relatively frequent in sentinel lymph nodes, likely secondary to the previous biopsy in the draining field (personal experience). Occasionaly these cells may present an interpretation challenge, but they can usually be interpreted as benign [6]. To avoid this interference by HMB45 and its lower sensitivity in general, HMB45 was not incorporated in the cocktail.

Out of 188 sections immunostained with the cocktail and interpreted by all five pathologists collectively, 41 were positive for melanoma micrometastases (Figure $2 \mathrm{~A}$ and $2 \mathrm{~B})$. Az values amongst the five pathologists interpreting these sections independently did not show statistically significant differences, demonstrating excellent interobserver agreement with the cocktail (Table 2). None 
of the 81 sections of mammary carcinoma sentinel lymph nodes showed immunoreactivity for the cocktail in any of the non-melanoma cells (Figure 2C) and were interpreted as negative for melanoma micrometastases by all five observers.

The cocktail showed favorable detection rate for melanoma micrometastases as compared to MART- 1 and MelanA alone. Of 78 sections, 25 sections were positive for melanoma micrometastases with the cocktail. However, only 21 were positive with MART-1 and Melan-A individually. Thus, MART-1 and Melan-A could not individually detect melanoma micrometastases in 16\% (4/25) of slices that were positive for melanoma micrometastases with the cocktail. As expected, combining a monoclonal antibody to tyrosinase with MART- 1 and Melan-A in the cocktail increased the spectrum to encompass three epitopes of two melanoma antigens (MART-1/Melan-A and tyrosinase). If indicated in the future, additional monoclonal antibodies showing immunostaining patterns in sentinel lymph nodes that are similar to these three antibodies which also posess high specificity for melanoma may be added to this cocktail, by widening the spectrum for many melanoma specific antigen epitopes. However, care should be taken to not include any antibody that may immunostain other non-melanoma cells in the sentinel lymph nodes. Otherwise, such antibodies may contribute to the deterioration in the specificity of the cocktail compromising its application for evaluation of the sentinel lymph nodes of melanoma.

During the standardization, all 35 melanomas (including one spindle cell melanoma and one clear cell melanoma) were immunoreactive with 'the cocktail'. Non-immunoreactivity of special variants of melanoma to various melanoma immunomarkers is a challenge. During interpretation of sentinel lymph nodes, the diagnosis of melanoma is known and the aim is to detect the melanoma micrometastases of the known primary. If the sentinel lymph nodes of special variants of melanoma are negative by 'the cocktail', it would be prudent to confirm the immunoreactivity of the primary lesion for 'the cocktail'. If the primary melanoma is non-immunoreactive for 'the cocktail', the negative interpretation of the sentinel lymph nodes with cocktail in this situation may be confirmed with other suitable immunomarker to which the primary melanoma is immunoreactive. The cocktail was not compared with S-100 in this study, as its role in evaluation of sentinel lymph nodes of melanoma was already demonstrated to be unreliable even in comparison to MART-1 and Melan-A individually in the previous study [6]. We did not compare tyrosinase alone with the cocktail because the questionable contribution of such a study would not be offset by the additional cost involved. Published studies do not report $100 \%$ immunostaining with anti-tyrosinase antibody (T311). Tyrosinase alone or any other immunomarker used individually, would be less sensitive than a cocktail. To evaluate the exact impact of the cocktail in comparison to all of its component immunomarkers individually for evaluation of sentinel lymph nodes, additional studies dedicated to comparison of individual component immunomarkers with the cocktail may be called for.

Capsular nevi in the form of a few cohesive cocktail immunoreactive spindle cells with bland elongated nuclei (Figure 3$)$ were observed in 5\% (9/188) of sections (10\% $(9 / 88)$ of sentinel lymph nodes and $21 \%(5 / 24)$ of cases. As compared to this, none of the 81 slices of sentinel lymph nodes from 30 cases of mammary carcinoma showed capsular melanocytic nevi. The sentinel lymph nodes of melanocytic nevi were from different sites and none were from the axillary region. The exact explanation for this difference in the prevalence of capsular nevi could not be deciphered and further evaluation is in process. The association of higher frequency of capsular melanocytic nevi with congenital nevi [23] is one possibility. In our study, except for the difference in their regional variation, another possible explanation is the cytokine effect of the melanoma draining to the sentinel lymph node. Hypothetically, an association between the higher prevalence of melanocytic capsular nevi and the predisposition for melanoma may be likely. Although, these melanocytic capsular nevi appear benign morphologically, a low probability that they may represent subtle metastases was also considered. Further evaluation of these and other possibilities in larger series is recommended.

Using immunohistochemistry, Yu et al [8] reported capsular benign melanocytic nevi in $4 \%(9 / 235)$ of sentinel lymph nodes of melanoma from $9 \%(8 / 94)$ cases (some cases had more than one sentinel lymph node). Capsular melanocytic nevi appear to be the most significant benign lesion that could pose a diagnostic challenge in the interpretation of melanoma micrometastases in sentinel lymph nodes. For similar reasons, non-in-situ molecular techniques, such as RT-PCR $[6,8,24-29]$ would have higher tendencies for false positive results. Morphology appears to be the single most important tool facilitating their correct interpretation [6]. Other approaches, including the observation that capsular nevi are non-immunoreactive or weakly immunoreactive for HMB45, have been suggested for differentiating capsular nevi from melanoma micrometastases [21]. However, it is important to note that a significant number of melanomas are also non-immunoreactive for HMB45. Thus, strong immunoreactivity for HMB45 rules out capsular nevus, but nonimmunoreactivity may not be contributory. 
Rather than immunostaining three adjacent sections with individual antibodies in the cocktail, only one slide is required which leads to cost savings. Although, the cost of the three antibodies used in the cocktail has to be incurred, the material and labor cost savings for one instead of three slides is obvious. For proper sampling, some studies recommend the evaluation of three levels at $200 \mu$ intervals [30]. In that case, if each of the three antibodies in the cocktail had to be used individually, it would require the immunostaining, interpreting, and correlating of nine sections. In contrast to this, the cocktail requires only three sections, each at intervals of $200 \mu$. Thus, use of the cocktail in concert with any protocol with reference to number of sections to be evaluated and proportion of sentinel lymph node to be sampled [30,31] would achieve obvious cost saving.

The most ideal positive control would be the sausage of three controls immunoreactive individually for one of the antibodies but non-immunoreactive for other two respective antibodies in the cocktail. However, this is a difficult positive control to get. The positive control used by us showed subtle differences when immunostained with each individual antibody in the cocktail with some areas slightly darker than others. However, when the same control was immunostained with the cocktail, the entire control showed relatively homogeneous immunostaining. The change in this immunostaining pattern would suggest the deterioration of one of the component which can be correlated with the same control immunostained with the individual antibodies. In addition, another precaution taken by us was that the cocktail was prepared after periodically evaluating individual antibodies with the positive control. This later approach is the best alternative.

\section{Conclusions}

It is possible to use a combination of monoclonal antibodies to MART-1, Melan-A, and Tyrosinase as a single cocktail reagent on one glass slide in contrast to three individual antibodies on three slides. The cocktail facilitated easy detection and interpretation of melanoma micrometastases in sentinel lymph nodes with reproducible interobserver results. There was favorable increase in the detection rate of melanoma micrometastases in sentinel lymph nodes as compared to MART-1 and Melan-A individually. Furthermore, this approach leads to obvious cost savings and simplifies the interpretation by reducing the number of slides to be evaluated.

\section{List of abbreviations}

AJCC, American Joint Committee on Cancer; Az value, area under ROC curves; DAB, Diaminobenzidine Hydrochloride; ROC curve, Receiver operating characteristic curve; S-100, S-100 protein.

\section{Competing interests}

None declared.

\section{Authors' contributions}

VS conceived, designed, carried out the entire study in addition to the standardization of MCW melanoma cocktail protocol and preparation of manuscript. DQ participated in the design of the study and assisted in data collection. CC participated in the design of the study and performed the statistical analysis. RNR, SA, BK, JM, and RK all participated in its design and coordination. GD performed the immunohistochemical staining including standardization of the MCW melanoma cocktail with VS. All authors read and approved the final manuscript.

\section{Acknowledgments}

Authors thank Ms. Patsy Gill for her secretarial assistance during the study. We also thank Dan Eastwood, MS for the statistical analysis of the data. Our thanks to Carl Becker, MD and Urias Almagro, MD for critically reviewing the manuscript. (This study was presented in part as a platform at The 91 st Annual Meeting of United States and Canadian Academy of Pathology, Feb 23-March I, 2002, Chicago, IL. [Mod Pathol 2002; 15: I A-368A. Abstract no. 450])

\section{References}

I. Crowley $N$ The case against elective lymphadenectomy Surg Clin North Am 1992, I:223-46

2. Heller R, Becker J, Wasselle J, Baekey P, Cruse W and Wells K Detection of submicroscopic lymph node metastases in patients with melanoma Arch Surg 199|, | 26: |455-60

3. Miliotes G, Albertini J, Berman C, Heller R, Messina J, Glass F, Cruse W, Rapaport D, Puleo C, Fenske N, Petsoglou C, Deconti R, Lyman $G$ and Reintgen $D$ The tumor biology of melanoma nodal metastases Am Surg 1996, 62:8I-88

4. Morton D, Wen D-R, Wong J, Economou JS, Cagle LA and Storm FK Technical details of intraoperative lymphatic mapping for early stage melanoma Arch Surg 1992, 127:392-9

5. Reintgen D, Cruse CW, Wells K, Berman C, Fenske N and Glass F The orderly progression of melanoma nodal metastases Ann Surg 1994, 220:759-67

6. Shidham VB, Qi D, Acker S, Kampalath B, Chang C, George V and Komorowski $R$ Evaluation of micrometastases in sentinel lymph nodes of cutaneous melanoma: Higher diagnostic accuracy with Melan-A and MART-I compared to S- 100 protein and HMB-45 Am J Surg Pathol 200I, 25: 1039-46

7. Tanabe $K$ and Reintgen $D$ The role of sentinel lymph node mapping for melanoma Adv Surg 1998, 31:79-103

8. Yu LL, Flotte TJ, Tanabe KK, Gadd MA, Cosimi AB, Sober AJ, Mihm $M C$ and Duncan LM Detection of microscopic melanoma metastases in sentinel lymph nodes Cancer 1999, 86:617-27

9. Brady MS and Coit DG Sentinel lymph node evaluation in melanoma Arch Dermatol 1997, 133:1014-20

10. Gershenwald JE, Colome MI, Lee JE, Mansfield PF, Tseng C and Lee JJ Patterns of recurrence following a negative sentinel lymph node biopsy in $\mathbf{2 4 3}$ patients with stage I or II melanoma Clin Oncol 1998, 16:2253-60

II. Reintgen DS, Cruse CW, Glass F and Fenske N In support of sentinel node biopsy as a standard of care for patients with malignant melanoma Dermatol Surg 2000, 26:1070-2

12. Qi D, Acker S, Kampalath B, Chang C, Komorowski R and Shidham VB Detection of Melanoma Micrometastases In Sentinel Lymph Nodes: Evaluation Of MART-I and Melan-A In Comparison To Traditionally Used Immunomarkers Am J Clin Pathol 2000, I | 4:629-59 Abstract no.-8

13. Jungbluth AA, Iverson K, Coplan K, Kolb D, Stockert E, Chen YT, Old $L$ J and Busam $K$ T3I I - an anti-tyrosinase monoclonal antibody for the detection of melanocytic lesions in paraffin embedded tissues Pathol Res Pract 2000, 196(4):235-42 
14. Shidham VB, Komorowski RA, Rao RN, Acker SM, Chang CC, Kampalath BN, Dawson G, Machhi JK and Qi DY Cocktail of three antibodies for the detection of micrometastases in sentinel lymph nodes of melanoma Mod Pathol 2002, I5:IA-368A Abstract no. 450

15. Beck JR and Shutz EK The use of relative operative characteristic (ROC) curves in test performance evaluation Arch Pathol Lab Med 1986, I I 0:13-20

16. McNeil BJ and Hanley JA Statistical approaches to the analysis of receiver operating characteristic (ROC) curves Med Decis Making 1984, 4:137-50

17. Raab SS Diagnostic accuracy in cytopathology Diagn Cytopathol 1994, 10:68-75

18. Raab SS, Thomas PA, Lenel JC, Bottles K, Fitzsimmons KM, Zaleski MS, Wittchow RJ, McPhaul LW, Slagel DD and Cohen MB Pathology and probability. Likelihood ratios and receiver operating characteristic curves in the interpretation of bronchial brush specimens Am J Clin Pathol 1995, I03:588-93

19. Shidham VB, Gupta D, Galindo L, Haber M, Grotkowski C, Edmonds $P$, Subichin S], George V and England I Intraoperative scrape cytology: Comparison with frozen section using probabilistic statistical method Diag Cytopathol 2000, 23:134-39

20. DeLong ER, DeLong DM and Clarke-Pearson DL Comparing the areas under two or more correlated receiver operating characteristic curves: A nonparametric approach Biometrics 1988, 44:837-45

21. Cochran AJ Surgical pathology remains pivotal in the evaluation of 'sentinel' lymph nodes Am J Surg Pathol 1999, 23 10:1 16972

22. Miettinen M, Fernandez M, Franssila K, Gatalica Z, Lasota J and Sarlomo-Rikala $M$ Microphthalmia transcription factor in the immunohistochemical diagnosis of metastatic melanoma: comparison with four other melanoma markers Am J Surg Pathol 200I, 25:205-II

23. Fontaine $D$, Parkhill $W$, Greer $W$ and Walsh $N$ Nevus cells in lymph nodes: an association with congenital cutaneous nevi Am J Dermatopathol 2002, 24: I-5

24. Blaheta HJ, Ellwanger U, Schittek B, Sotlar K, MacZey E, Breuninger $\mathrm{H}$, Thelen MH, Bueltmann B, Rassner $\mathrm{G}$ and Garbe $\mathrm{C}$ Examination of regional lymph nodes by sentinel node biopsy and molecular analysis provides new staging facilities in primary cutaneous melanoma / Invest Dermatol 2000, I I 4:637-42

25. Blaheta HJ, Paul T, Sotlar K, Maczey E, Schittek B, Paul A, Moehrle M, Breuninger $H$, Bueltmann B, Rassner $G$ and Garbe $C$ Detection of melanoma cells in sentinel lymph nodes, bone marrow and peripheral blood by a reverse transcription-polymerase chain reaction assay in patients with primary cutaneous melanoma: association with Breslow's tumour thickness $\mathrm{Br}$ J Dermatol 200।, I 45:195-202

26. Ghossein $\mathrm{R}$ and Rosai J Polymerase chain reaction in the detection of micrometastases and circulating tumor cells Cancer 1996, 78:10-6

27. Messina JL, Glass LF, Cruse CW, Berman C, Ku NK and Reintgen DS Pathologic examination of the sentinel lymph node in malignant melanoma Am J Surg Pathol 1999, 23:686-90

28. Shivers SC, Wang X, Li W, Joseph E, Messina J, Glass LF, DeConti R, Cruse CW, Berman C, Fenske NA, Lyman GH and Reintgen DS Molecular staging of malignant melanoma: correlation with clinical outcome $J A M A|998,280:| 4 \mid 0-5$

29. Sung J, Li W, Shivers $S$ and Reintgen D Molecular analysis in evaluating the sentinel node in malignant melanoma Ann Surg Oncol 200I, 8(9 Suppl):29S-30S

30. Treseler PA and Tauchi PS Sentinel lymph nodes in human solid cancer. Pathologic analysis of the sentinel lymph node Surg Clin North Am 2000, 80:1695-19

31. Farshid G, Pradhan M, Kollias J and Gill PG Computer simulations of lymph node metastasis for optimizing the pathologic examination of sentinel lymph nodes in patients with breast carcinoma Cancer 2000, 89:2527-37

\section{Pre-publication history}

The pre-publication history for this paper can be accessed here: http://www.biomedcentral.com/1471-2407/3/15/prepub

Publish with Bio Med Central and every scientist can read your work free of charge

"BioMed Central will be the most significant development for disseminating the results of biomedical research in our lifetime. "

Sir Paul Nurse, Cancer Research UK

Your research papers will be:

- available free of charge to the entire biomedical community

- peer reviewed and published immediately upon acceptance

- cited in PubMed and archived on PubMed Central

- yours - you keep the copyright
BioMedcentral 